\title{
Survey of Expert Systems and the Cognitive Approaches towards an Effective Tutoring System
}

\author{
D.K. Chaturvedi \\ Dayalbagh Educational Institute \\ Department Of Electrical Engineering \\ Agra, India 282005
}

\author{
Atul Prakash Prajapati \\ Dayalbagh Educational Institute \\ Department Of Electrical Engineering \\ Agra, India 282005
}

\begin{abstract}
This paper presents a survey of Expert Tutoring System (ETS), designed for the improvement of teaching pedagogy. It talks about the flaws and designing issues that may occur in designing the expert tutoring system, and also suggests the cognitive approach for building a robust tutoring system. It describes the ill defined domains, case based reasoning, and the system approach for the designing of a cognitive tutoring system.
\end{abstract}

\section{General Terms}

Expert Tutoring Systems (ETS), Conventional ETS, Cognitive ETS, Pedagogy

\section{Keywords}

Expert Tutoring Systems (ETS), Poorly Defined Domains, Case Based Reasoning (CBR), Cognitive Approach

\section{INTRODUCTION}

Designing a cognitive tutoring system is itself a tedious task, for this various approaches (like machine learning, well defined domain, evidence (or fact) based reasoning approaches, robust knowledge-base) play an important role. These approaches come under the area of artificial intelligence, which is an emerging field of computer science.

Researchers are working hardly since 1950 in this area. But even after 50 years of research, they are not able to deign an expert tutoring system that is social and able to dispose its duties as desired in the real world environment.

The reason behind the failure is, the techniques that is used in designing of traditional systems. These techniques generally follow the rule based approach, here the knowledge-base consists of set of hard coded rules, that is of fixed in nature and usually do not change over the time. Here one trains one's agent in abstraction (in idle circumstances), that's why the systems can not perform better in the real world environment. So for the improvement, one should use those techniques that train their agents in real world environment rather in abstraction.

This paper presents a survey of present/past expert tutoring systems, their virtues and drawbacks. It proposes the cognitive approach for designing cognitive intelligent tutoring systems (ITS). This approach is one of the best approaches for the development of intelligent systems, and it guarantees that a cognitive system can perform better in real world environment than ITS systems. This paper discusses about review of expert tutoring systems (ETS), well defined versus ill defined domains, case based reasoning (A CBR Approach), cognitive approach for building an effective tutoring system.

\section{LAYOUT OF A CONVENTIONAL ETS SYSTEM}

Over the past one decade, ETS systems are widely accepted and used as a learning tool for the students and also for assisting teachers in their teachings. Many ETS systems have been designed in various fields like teaching mathematical concepts, basic physics concepts, programming techniques, gaming concepts, as a simulation tool etc. So for the proper understanding about the basic functioning of an ETS system, one should study each and individual component of an ETS system.

If we talk about the taxonomy of an expert tutoring system, then an expert system is a generalization of the following components.

\subsection{Knowledge Base}

Knowledge-base is the back bone of any expert system. It stores the previous experiences (features of an object), that will be used further for inferencing. Knowledge-base differs with database, as it is action oriented. So one should be very precise while designing a knowledge-base. It should be indexed and unambiguous. Basically it should have all the features of a well defined domain.

\subsection{Student Module}

Every ETS system should have a student module. This module deals with the students, as student can learn new concepts and put their queries also. It should be user friendly with required level of abstraction. A student module is linked with the knowledge base and with the teacher module for inferencing.

\subsection{Teacher Module}

Analogous to the knowledge-base this module is also a main component of an ETS system. It defines the strategies for building a robust knowledge-base. Here a human teacher teaches to an ETS system, by providing various strategies (like it may include images, videos, or references) to teach effectively a particular concept. Here a human tutor makes a set of extracted features, stores 


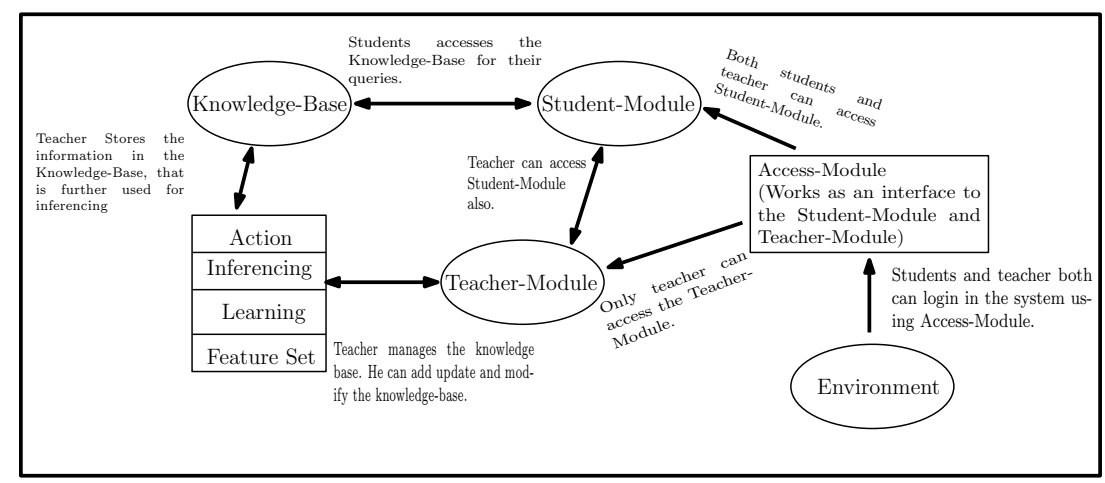

Fig. 1. Schematic diagram of ETS system

them in knowledge-base, and also defines what actions it has to take whenever a similar problem encounters.

\subsection{Output Module OR Access Module}

It is a front-end of an ETS system. It works as an interface that is used to provide accessibility of the system to it's users. It integrates all the modules and provides an appropriate level of abstraction to make an ETS system user friendly. It provides an input module to the system and an output module from the system. Input and output to and from the system may be of any form like it may include multimedia, graphics, text etc.

\section{DIFFERENT EXPERT SYSTEMS FOR ASSISTING AND IMPROVING TEACHING PEDAGOGY}

Over the past two decades lots of expert systems have been designed for the improvement of teaching pedagogy. This paper presents a survey of these expert systems. In the year 1979, Tim o shea [51] proposed a self improving expert teaching system. He proposed that if any system has more than one programs to teach the same concept, then it would be an effective way to make the clear understanding about the topic. As teachers do sometimes, they use to teach the same thing by using different examples. For achieving this functionality he included different alternative programs in his system. In the year 1984, Gerhard et al. [16] proposed an online help system "Active Help System". In their paper they suggested that, as the functionality increases a system becomes more complex to deal with it. So there will be a need of a help module. The passive help system that is present inside any complex system to guide the users is of very less use, because of the increased complexity. So they suggested a good user friendly on-line help system that may solve the problem. Further in 1984, Williom et al. [9] proposed a system that can help in debugging PROLOG programs. They explained how the preventive way of programming plays an important role in developing a bug free program. For inculcating this programming style in PROLOG programmers, they proposed some debugging tips. These techniques can help a PROLOG programmer in writing a bug free program. In the year 1986, Nigan Bayazit [4] proposed a model designed to teach CAD techniques to the students of Istanbul Technical University (ITU). This model has two parts, first part is designed for the students and second part is designed for teachers and staff members of the university. In the year 1986, Massimo Gallanti et al. [18] presented a paper, in this paper they explained the importance of expert system ap- proach for designing an intelligent decision aid system. They also presented a case study in which they took an example of a thermal power plant, and proved the significance of expert system approach in decision making process. In the year 1987, R. S. Perez et al. [45] had proposed a simulation tool that was used to provide an effective training to the military personals by using computers. It was relatively cheaper, as the software can be installed in any personal computer. No additional hardware was required for this type of training. They also declared that this type of training was more effective, as it was uniform to all and used to provide an animated environment that was preferred by the trainees. In the year 1987, W. Feurzeig [60] proposed that over the past one decade researchers have preferred more to the intelligent CAI (Computer Aided Instruction) intelligent tutoring systems than the traditional ITS systems for providing complex training. They also presented that systems like TRIO, SOPHIE, and STEAMER was very useful and powerful systems for providing the complex training in various fields like plant operation and electronic troubleshooting. Further in the year 1987, Emrah Orhun [43] proposed the importance of CAI (Computer-Assisted Instructions) over the traditional way of classroom teaching. As CAI teaching method is superior, it is more effective, less costly and can pay more attention rather than classroom teaching. In the year 1987, J. G. Neal et al. [25] presented a NLU (Natural Language Understanding) system. They designed this system to teach natural languages to the non native speakers and to the poor language speakers. For this they used an AI system that uses rule based approach and for inferencing it uses the knowledge base. This system improves and provides NL understanding clearly to its users. In the year 1987, L. H. Nawrocki [30] presented an ICAI (Computer assisted Instructional Model) system for providing maintenance training. This system was designed with the joint cooperation of industrial and military department for proving training to their personals. In the year 1987, A. Brebner et al. [2] proposed a computer based instruction terminal (Caiter). This terminal system proposed that, if one incorporates multimedia approaches with computer aided instructions, then it would be more effective for the trainees. In the year 1987, E. Dmc. Williams et al. [13] proposed a CBI (Computer Based Instruction) system, sponsored by naval research wing of USA. The purpose of this system was to provide some intelligence to the existing CBI system, so that it can be used to provide training effectively to the military personals. As they have to invest a lot of time and money to train their soldiers. They found that this technical improvement converted a CBI system in an effective and interesting tool for providing training. In the year 1987, Kazuhisa Kawai et al. [26] proposed a new 
ICAI (Intelligent Computer-Assisted Instruction) system. This system had two main modules expertise and the student module. They used inductive inferencing for student module, and both modules were designed using PROLOG language. These two modules were built independently so they were domain independent also. In the year 1987, David G. Ullman et al. [56] illustrated the role of CAD (Computer Aided Design) and knowledge-base based expert systems in machine designing process. They also stated that these techniques will be the future of complex machine designing. They recorded the verbalization of the experts while designing complex systems in the tutor's knowledge-base, so that it may be used as a helping tool in future. In the year 1988, Joachim Wedekind [59] proposed the importance of simulation tool to teach modelling. As this is a cost effective and can cover all the aspects of modelling. It provides a virtual environment and one can create all types of faults and defects without affecting the actual machines. In the year 1988, D. Partridge, [44] explained two approaches for designing an intelligent systems, Symbolic and connectionist approach. In the year 1988, Randall et al. [61] explained that the expert systems are not defined properly yet, the present definitions are inadequate. They defined ES systems as a communication tool, and tried to find out the factors that affect the applicability of an ES system in an enterprise, so that the data loss can be minimized. In the year 1988, Dario [20] explained the importance of LISP (a programming language) for designing prototype of complex software. Over the past one decade it has been observed that the performance of the LISP systems was increased over the systems that was designed using traditional programming languages. In a case study presented by Dario, he stated that the systems that was designed using LISP takes less development time compared to other programming languages. To prove this fact he presented a tutoring system for teaching Chinese language to the beginners, which was designed in less than three weeks. This tutoring system had two main components student module and knowledge-base, and all the data structures for the knowledge-base was designed using LISP language and existing tools. In the year 1989, Nakhoon et al. [27] presented an ITS (Intelligent Tutoring System) to teach the relation in between circulatory physiology and stabilized blood pressure systems. It is used to provide an simulation environment for creating various faults, so that students can learn and remove their misconceptions. This system provides an animated environment to improve learning in among of the students during diagnosis. It generates a bug report by taking inputs from the students, and produces an output set using natural languages. In the year 1989, Joel et al. [40] developed a PPT (Patho-physiology Tutor) to teach cardiovascular physiology concepts by providing a hypothetical environment generated by this tutor. It was designed using turbo PROLOG language and rule based approach was used for designing its knowledge-base. It provides a simple menu based environment for input and provides small set of tutorials as an output to teach the basic concepts. In the year 1989, Mohammed et al. [23] designed a Tutoring Rule Authoring System (TRAS), for the teachers and other programming language personals to develop rules for the development of rule-based knowledge-base. It has two main modules TPT (Text to PROLOG Translator) and TRE (Tutoring Rule Editor). This rule editor module (TRE) was used to create rules and also with the help of this module one can change or completely remove any rule from the knowledge-base, and the TPT module was used to represent rules in a proper format so that it can run as a PROLOG program. In the 1989, Marilun et al. [32] designed a Quadratic Grapher ITS system, for graphical representation of quadratic equations. In the year 1989, Michael [5] presented an overview of MATHEPERT ITS system, that is designed for teaching basic calculus, trigonom- etry, and algebraic rules to the students. He also explained how this system generates explanations and error messages to an individual. Here error messages was generated by comparing the solutions provided by the user and the solutions that is present in its knowledge-base. In the year 1989, Janis et al. [7] presented the concepts of KBMS (Knowledge-Base Management System) and how it functions. In the year 1989, Ali Farghaly [15] proposed a new software MICALI (Modified Computer Assisted Language Instruction). He suggested that such expert software should be developed by using natural language processing tools, to make them user friendly. MICALI was a highly interactive software that was able to communicate with the students by using the knowledge defined in its knowledge-base. In the year 1990, Parvati Rajan et al.47] designed a tutor to teach basic trigonometric concepts to the students. In the year 1990 Gilles et al. [24] presented a survey of ITS systems designed to teach word techniques. They also proposed an ITS system "WORD-TUTOR" for teaching basic word concepts. In the year 1990, A. Benslimane et al. [6] proposed the concept of teaching plan for an ITS system. If an ITS system includes this module, it can provide table of contents also. The benefit of including this module to an ITS system was, now a student can check the list of contents. In the year 1990, Daniel M. Russle [49] proposed an ITS system named "Alexandria", which was designed to teach historical events of third century B.C. to the students. In the year 1991, Yoon Hee lee et al. 29 proposed a spelling checking and correcting program for an ITS system that was designed for medical students. The purpose was to improve inferencing power of the ITS system, as it can check and correct the wrong spellings, can add missing characters, and can perform character substitution. So that an ITS system can receive the accurate information about the context, and accordingly can produce better outcome. In the year 1991, H. Kindler et al. [28] presented an ITS system named "RADES" designed for the management of accidentaly irradiated persons. In the year 1991, M. Quafafou et al. [46] proposed a simulation tool for the mining engineering students. This tool provides an animated environment for teaching simulation of exploration drillings. It was developed in $\mathrm{C}++$ language. In the year 1991, Luigia et al. [3] proposed a multi agent ITS system. For representation of knowledge in knowledge-base they used first order predicate calculus. Here knowledge base of agents are kept with the agent itself, no common knowledge-base exists there. For reasoning agent can check its knowledge-base as well as other agent's knowledge-base also (a societal way of solving problems). In the year 1992, Albert et al. [11] proposed a ACT tutor that teaches programming concepts to the students. It contains hundreds of rules and it solves the exercise step by step with the students. Finally it produces the student's performance report, that will be provided as an input to the teacher module for the improvement of student's performance. In the year 1992, Eric [22] proposed an ITS system named "SIFT tutor" (self improving fractions tutor). The basic idea behind to develop this tool was to improve the performance of ITS systems. This SIFT tutor learns and improves its knowledge while interacting with the students. It used Dempster-Shafer theory of evidence for updating its knowledge-base. In the year 1992, Jean et al. [39] developed a ITS system named "GRACE" by collaborating with NYNEX Science and Technology for teaching COBOL language. Finally, they described that the main drawback of their system was to bring it from lab to classroom every day, that was a very tedious task. In the year 1993, Haider et al. [48] proposed an ITS system "DISCOVER" for teaching programming concepts. This system was capable of monitoring student performance also. In the year 1993, Vijay et al. [58] developed a computer based instructional system "Turbinia-Vyasa", for providing training on the 
trouble shooting marine. It was developed on Apple machintosh systems. Here Vyasa was an intelligent tutor and Turbinia provided simulation environment. In the year 1993, R. Macura et al. [36] proposed an ITS system to teach radiology concepts by providing various cases for diagnosis. Here they proposed a database having various radiology concepts integrated with images. For updating database a radiologist analyses the images stored in archive, then apply his knowledge for searching related images on the web, and stores new images in the database. So that it's database can be augmented, that would help in teaching radiology concepts effectively. This system consists of three modules, Atlas, Case Retrieval, Teach-me. In the year 1993, M. Joao [33] proposed an ITS system "LABEL" for providing intelligent learning environment by teaching the concepts of electrical circuits. The purpose for designing this system was to contribute in the field of education as well as in the field of research also. In the year 1994, Robert et al. [55] proposed rules for the development of an effective ISD (Automated Instructional System Development) system. The purpose of this paper was to improve the designing of conventional ISD systems, as new technologies like cognitive science, machine learning have been introduced. In the year 1994, Kenneth [53] proposed a system "DISCOURSE". This system provides the information about different tools that is required for designing a multimedia based simulation environment for effective learning. The basic drawback present in conventional simulators is that, it provides a common integrated environment for simulation and as well as for showing the results also. Because of that students face problems in relating the modules. This system proposed the separate environment for these modules. Further the outcome of result module can be stored in knowledge-base for designing pedagogical strategies. In the year 1995, Robert [54] described the fourth generation ISD systems. In the year 1995, Jeroen et al. [57] proposed a model "FILM's" (Fuzzy Logic Instructional Models) that has applied in an ITS system "CASCO" for automatically generating assignments for the students. This paper describes the architecture of CASCO and the process of creating assignments dynamically provided by FILM. In the year 1996, Michael et al. [50] presented a conventional language learning tool for helping teachers. As this tool can create tutorials, graphical representation of the objects. In the year 1996, Jon et al. [14] suggested a new approach CBR "case based reasoning approach" for the development of an effective ITS system. A case based instructional planner had also been proposed in integration with conventional ITS systems for the development of a robust hybrid system. So that this system can also generate teaching plans for the teachers. In the year 1996, Chee-kit et al. [31] designed a tutor "WORDMATH" for teaching word concepts to the children of age group 9-12 years in the Singapore primary school. In the year 1998, Mary et al. [38] proposed some new strategies for the improvement of teaching skills in an existing algebra tutor "PAT1". Further in the year 2000, Albert [10] presented the case study of an algebra tutor "Algebra II cognitive tutor". In the year 2004, Eva et al. [41] presented a web based tutor "TAPLI", that was designed to teach the concepts of linear programming. In the year 2005, Carbolina et al. [21] proposed the concept of multi agents for modelling student module. They proposed four types of agents based on their working, CLA agent (Case Learner Agent), TA (Tutor Agent), AA (Adaptation Agent), and OA (Orientator Agent). Each agent was designed for a particular task, like TA agent was designed to select teaching strategy, OA agent reads the psychological behaviour of the student, that helps in providing more personalized teaching. In the year 2007, Xin et al. [34] presented a comparative study of Expert and Non-Expert tutoring system, and proved by providing various facts that expert systems are superior to non- expert systems. In an expert system student can talk to the tutor by using natural languages, that makes these systems user friendly. In the year 2010, Philippe [17] proposed that present ITS systems are facing lots of problems, because of the wrong specifications used for designing them. So he proposed a Hybrid approach for designing effective tutoring systems. In this he used the concept of well defined domains and applied new approaches for data mining. In the year 2012, D.K.Chaturvedi [8] proposed an on-line simulation tool for virtual power lab. This simulation tool provides an on-line simulation environment for teaching power lab concepts. In the year 2013, Ivanova. M [42] proposed the concept of facial and gesture recognition method for an ITS system. If one applies these techniques in their system, it will act something like human tutor. As human tutor can identify by checking the gesture and facial expression of a student, that whether they are getting the contents or not. So this approach can be useful in designing effective tutoring systems. In the year 2014, Steif et al.[52] proposed a cognitive tutor approach for solving truss problems in statics. They explained that in traditional learning, teachers give assignments to their students for evaluating student's performance. But this traditional approach is not so effective as timely and personalized checking of all the assignments are not possible each time. So they suggested an ITS system that maintains a set of solutions to the problems, now students can interact with this tutor for finding the solutions to their problems. This tutor also proposed a feed-back mechanism for evaluating their performance. In the year 2014, Malick et al. [37] proposed a tutor "Carrom-Tutor" for teaching skills of a game named "carrom". This tutor was designed with the help of BLENDER (3-D animation suite). They also presented a case study of carrom king (an on-line game), and proved that their carrom tutor was superior to the carrom king as it provides easy tutorials and new strategies to play this game. Further in the year 2015, Corbi et al. [12] proposed a rule based approach LIME for modelling the database of PSLC cognitive tutor provided by (Pittsburg Science Learning Centre), and Carnegie Mellon University helped in providing timely on-demand feed-back for the construction of error free and effective system.

\section{A NOVEL APPROACH TO FILL THE GAP OF CONVENTIONAL TUTORING SYSTEMS}

Following sections define the approaches for designing a robust cognitive tutor.

\subsection{Features of a robust cognitive tutoring system}

One can categorize any domain in two parts [35], ill defined domains and well defined domains. Many philosophers had proposed various definitions for these domains. Generally these definitions lie in between artificial intelligence theory and decision making problems. This paper follows the artificial intelligence approach for defining these domains. According to John Mc Carthy and Marvin Minsky "A well defined domain is like a knowledge-Base, which can provide unambiguous and robust solutions to any particular problem or when one should use a particular solution for a problem". Well defined domains are typically quantitative. For designing these domains human tutors feed propositional rules in its knowledge-base. These rules should be unambiguous for the better inferencing.

Antithesis to this, most of the tutoring systems have ill defined domains. Typically the reason is ambiguous solutions to the problems and non adaptive nature of knowledge-base. That result in poor inferencing. 
Following are some key points that plays an important role in the development of well defined domains.

- It should have robust planning strategy while dealing with new objects.

- It should have proper and accurate way of data acquisition methods.

—It should have an adaptive knowledge-base.

-It should have proper structuring of the learning in its knowledge-base.

\subsection{Case Based Reasoning approach (A CBR Approach)}

This approach is used in making learning strategies and providing reasoning capability to any ITS system. It is an incremental way of learning (adaptive in nature), that is further used for inferencing. This problem solving method is analogous to the way as humans do. They enhances their learnings based on their experiences and apply them back to solve new problems. Similarly a CBR system treats each problem as a case and stores these cases in its knowledge-base for building a solution space, that is further used for inferencing. A CBR is a sub-field of machine learning approach and proposes a cyclic way for problem solving. It analyses the new problem, finds similar cases in its knowledge-base, combines the solution, produces the result, and updates the knowledge-base.

-Retrieve.

-Reuse.

-Revise.

-Retain.

According to Agnar [1] following are some terms that are similar to CBR approach.

(1) Example based reasoning: A solution space is constructed by the set of similar examples.

(2) Instance based reasoning: It is a specialization of example based reasoning.

(3) Memory based reasoning: Here cases (past problems) are stored in memory, and for inferencing and reasoning similar cases are searched in memory.

(4) Analogy based reasoning: According to Dedre [19] It is a kind of mapping in between objects, for defining unknown objects. Suppose there exist an analogy "Some A is (like) B", it defines a relation in between $\mathrm{A}$ and $\mathrm{B}$. Here $\mathrm{A}$ is the target object (unknown object) and $\mathrm{B}$ is a base object (known object). so an analogy is a way that relates unknown objects to the known objects to make clear understanding about the unknown objects.

\subsection{Cognitive approach}

To be an intelligent system only few abilities like recognition and decision making is required. But to perform better in the real world environment, or to be a cognitive system. It should have some additional set of capabilities. Following are some properties that a cognitive system should have.

4.3.1 Recognition capability. Recognizing something is the part of feature extraction, and it should follow a proper sequence.

(1) Perception.

(2) Feature extraction.

(3) Inferencing.
(4) Categorization and Learning.

(5) Action.

4.3.2 Decision making capability. It is directly proportional to the learning, with the help of learning an agent can decide which alternative it should choose in among of available alternatives.

4.3.3 Perception and situation assessment capability. A cognitive agent should be able to correctly extract the set of features of an object in any environmental condition. For that it should use robust sensory data collection techniques, as external noises play a destructive role in perceiving things.

4.3.4 Prediction and monitoring capability. This is something similar to a human level intelligence. As a good driver can predict that after few kilo-meters, his car will be in need of refuelling. This type of ability can only be achieved by strong monitoring mechanism and experience.

4.3.5 Reasoning capability. Cognitive agent should have reasoning ability for producing more accurate results. For acquiring this capability, an agent should be able to establish relationships between the stored data in its knowledge base. It can use techniques like first order logic, production rules, neural networks for establishing relationship in between the objects, and knowledge about the reasoning can be heuristic. This is related to the learning. By using these techniques an agent can make new decisions and store them back in the knowledge base, or it can change previous beliefs also.

4.3.6 Interaction and communication capability. The cognitive agents should work in a society manner. As in a society the work is done by consulting other society members. Analogously the agents should communicate to each other for the best inferencing. Also for recognizing any object in a real word environment, memory is required. The communication in between or in among of agents may be in the form of query message, audio files, video files.

4.3.7 Learning and reflection capability. To create learning a cognitive agent should be able to extract features of an object and store them in a proper format in its knowledge-base. So that whenever it has to recognize any object it can consult to its knowledgebase.

\section{CONCLUSION}

Finally this paper presents a survey of ETS systems, that has been designed for the improvement of teaching or basically for assisting pedagogy. It talks about the ill defined domains and also proposes the concept of well defined domains, by which one can design a robust (that can perform better in real world environment) ETS systems. It talks about the CBR approach, this study of CBR approach inculcates the idea behind how one can provide intelligence and reasoning capability in an ETS system. Finally, the main component of this paper is cognitive approach. It suggests the basic techniques for designing an ETS system. A cognitive ETS system is far more superior to an intelligent system, as it consists of knowledgebase, and here the agents learn in the real world environment rather than in abstraction.

\section{ACKNOWLEDGEMENT}

I would like to thank Mr. Ashish Chandiok for his valuable support and timely suggestions. 


\section{REFERENCES}

[1] Agnar Aamodt. Case-based reasoning: Foundational issues, methodological variations, and system approaches. AI Communications, 7(1):39-59, 1994.

[2] A.Brebner, H.J Hallworth, and G.Mckinnon. Caiter: a computer based instruction terminal. Springer US, 1987.

[3] Luigia Carlucci Aiello, Daniele Nardi, and Marco Schaerf. Reasoning about reasoning in a meta-level architecture. Applied Intelligence, 1:55-67, 1991.

[4] Nigam Bayazit. An interactive model for computer aided design education and research on knowledge base of designers. Springer US, 1986.

[5] Michael J. Beeson. Logic and Computation in MATHPERT: An Expert System for Learning Mathematics. Springer US, 1989.

[6] A. Benslimane and C. F. Ducateau. An Expert System for the Elaboration of Teaching Plan Based on a Subject-Matter Model. Springer London, 1990.

[7] Janis A. Bubenko and Istvan P. Orci. Knowledge Base Management Systems: A Database View. Springer Berlin Heidelberg, 1989.

[8] Devendra Kumar Chaturvedi. Virtual power lab: A better opportunitie for learning. International journal of information technology and Application, 2, 2012.

[9] William F. Clocksim and Christopher S. Mellish. Debugging prolog programs. Springer US, 1984.

[10] Albert Corbett, Megan McLaughlin, K. Christine Scarpinatto, and William Hadley. Analyzing and Generating Mathematical Models: An Algebra II Cognitive Tutor Design Study. Springer Berlin Heidelberg, 2000.

[11] Albert T. Corbett and John R. Anderson. Student modeling and mastery learning in a computer-based programming tutor. Springer Berlin Heidelberg, 1992.

[12] Corbi and A. Burgos. Implementation of the recommendation model lime in cognitive and visual interactive tutors from pslc. Latin America Transactions, IEEE (Revista IEEE America Latina), 13(2):516-522, 2015.

[13] E.D.Mcwilliams and G.L.Richard. Portable, intelligent simulation for ASW training. Springer US, 1987.

[14] Jon A. Elorriaga and Isabel Fernndez-Castro. The HSIIP approach. An extension for a teacher's apprentice. Springer Berlin Heidelberg, 1996.

[15] Ali Farghaly. A model for intelligent computer assisted language instruction (micali). Computers and the Humanities, 23:235-250, 1989

[16] Gerhard Fischer, Andreas Lemke, and Thomas Schwab. Active help systems. Springer US, 1984.

[17] Philippe Fournier-Viger, Roger Nkambou, Engelbert Mephu Nguifo, and Andre Mayers. ITS in Ill-Defined Domains: Toward Hybrid Approaches. Springer Berlin Heidelberg, 2010.

[18] Massimo Gallonti and Giovanni Gwida. Intelligent decisions aids for process environments: An expert system approach. Springer US, 1986.

[19] Dedre Gentner. In Structure-mapping, A theoritical framework for analogy, volume 7, pages 155-170. Bolt beranek and newman inc., 1983.

[20] Dario Giuse. Lisp as a rapid prototyping environment: The chinese tutor. Lisp and symbolic computation, 1:165-184, 1988.
[21] Carolina Gonzalez, Juan C. Burguillo, and Martin Llamas Case-Based Student Modeling in Multi-agent Learning Environment. Springer Berlin Heidelberg, 2005.

[22] Eric Gutstein. Using expert tutor knowledge to design a SelfImproving intelligent tutoring system. Springer Berlin Heidelberg, 1992.

[23] Mohammed M. Haque, Allen A. Rovick, Joel A. Michael, and Martha Evens. Tutoring Rule Authoring System (TRAS). Springer Berlin Heidelberg, 1989.

[24] Gilles Imbeau, Gilles Gauthier, and Claude Frasson. Wordtutor: An intelligent tutoring system for teaching word processing. Springer Berlin Heidelberg, 1990.

[25] J.G.Neal and S.C.Shapiro. Knowledge based parsing Springer US, 1987.

[26] Kazuhisa Kawai, Riichiro Mizoguchi, Osamu Kakusho, and Junichi Tyoda. A framework for icai system based on inductive inference and logic progamming. New generation computing, 5:115-129, 1987.

[27] Nakhoon Kim, Martha Evens, Joel A. Michael, and Allen A. Rovick. Circsim-tutor: An intelligent tutoring system for circulatory physiology. Springer Berlin Heidelberg, 1989.

[28] H. Kindler, D. Densow, and T. M. Fliedner. RADES Medical Assistance System for the Management of Irradiated Persons. Springer Vienna, 1991.

[29] Yoon Hee Lee, Martha Evens, Joel A. Michael, and Allen A Rovick. Spelling correction for an intelligent tutoring system. Springer New York, 1991

[30] L.H.Nawrocki. An intelligent computer assisted instruction system for maintenance training. Springer US, 1987.

[31] Chee-Kit Looi and Boon Tee Tan. WORDMATH: A computerbased environment for learning word problem solving. Springer Berlin Heidelberg, 1996.

[32] Marilyn Loser and Barry Kurtz. Quadratic grapher: An intelligent tutoring system for graphing quadratic equations. Springer Berlin Heidelberg, 1989.

[33] M. Joo Loureiro. LABEL: An Intelligent Learning Environment (ILE) for Electric Circuits. Springer Berlin Heidelberg, 1993.

[34] Xin Lu, Barbara Di Eugenio, Trina C. Kershaw, Stellan Ohlsson, and Andrew Corrigan-Halpern. Expert vs. Non-expert Tutoring: Dialogue Moves, Interaction Patterns and Multiutterance Turns. Springer Berlin Heidelberg, 2007.

[35] Collin F. Lynch, Kevin D. Ashley, Vincent Aleven, and Niels Pinkwart. Intelligent tutoring systems for ill-defined domains. In Proceedings of the Workshop on Intelligent Tutoring Systems for Ill-Defined Domains, Taiwan, 2006.

[36] R. Macura, K. Macura, V. Toro, E. Binet, and J. Trueblood. Case-Based Tutor for Radiology. Springer Berlin Heidelberg, 1993.

[37] M. Malick, M. Katke, and S Iyer. CarromTutor: A Cognitive Apprenticeship Based Tutor for Carrom Skills and Strategies. Technology for Education (T4E), 2014 IEEE Sixth International Conference in Clappana, 2014.

[38] Mary A. Mark, Kenneth R. Koedinger, and William S. Hadley. Elaborating Models of Algebraic ExpressionWriting. Springer Berlin Heidelberg, 1998.

[39] Jean McKendree, Bob Radlinski, and Michael E. Atwood. The Grace Tutor: A qualified success. Springer Berlin Heidelberg, 1992. 
[40] Joel A. Michael, Mohammed M. Haque, Allen A. Rovick, and Martha Evens. The pathophysiology tutor: A first step towards a smart tutor. Springer Berlin Heidelberg, 1989.

[41] Eva Millan, Emilio Garcia-Hervas, Eduardo Guzman De los Riscos, Angel Rueda, and Jose Luis Perez de la Cruz. TAPLI: An Adaptive Web-Based Learning Environment for Linear Programming. Springer Berlin Heidelberg, 2004.

[42] M.Ivanova. Researching affective computing techniques for intelligent tutoring systems. Interactive Collaborative Learning (ICL), 2013 International Conference in Kazan, 2013.

[43] Emrah Orhun. Expert systems and intelligent computerassisted instruction. Springer US, 1987.

[44] D. Partridge and K. Paap. An introduction to learning. Artificial intelligence review, 2:79-101, 1988.

[45] R.S. Perez and R.J.Seidel. The use of intelligent authoring tools to enhance CBI in technical training. Springer US, 1987.

[46] M. Quafafou, O. Dubant, J. P. Rolley, and P. Prvot. The Design of a Simulator System for Educating Engineers. Springer Netherlands, 1991.

[47] Parvati Rajan, Pramod Patil, KSR Anjaneyulu, and P Srinivas. The trigonometry tutor. Springer Berlin Heidelberg, 1990.

[48] Haider Ramadhan and Benedict du Boulay. Programming Environments for Novices. Springer Berlin Heidelberg, 1993.

[49] Daniel M. Russell. Alexandria: A Learning Resources Management Architecture. Springer Berlin Heidelberg, 1990.

[50] Michael Schoelles and Henry Hamburger. Teacher-usable exercise design tools. Springer Berlin Heidelberg, 1996.

[51] Tim O Shea. A design for self improving teaching system. Springer US, 1979.

[52] P.S Steif, Fu Luoting, and L.B Kara. Computer tutors can address students learning to solve complex engineering problems. Frontiers in Education Conference (FIE), 2014 IEEE Madrid, 2014.

[53] Kenneth Tait. DISCOURSE: The Design and Production of Simulation-based Learning Environments. Springer Netherlands, 1994.

[54] Robert D. Tennyson. Instructional System Development: The Fourth Generation. Springer Berlin Heidelberg, 1995.

[55] Robert D. Tennyson and Klaus Breuer. ISD EXPERT: An Automated Approach to Instructional Design. Springer Berlin Heidelberg, 1994.

[56] David G. Ullman and Thomas A.Dietterich. Mechanical design methodology implications on future developments of computer-aided design and knowledge-based systems. Engineering with computers, 2:21-29, 1987.

[57] Jeroen J. G. van Merrinboer, Jaap Jan Luursema, Hans Kingma, Frans Houweling, and Arjen P. de Vries. Fuzzy Logic Instructional Models: The Dynamic Construction of Programming Assignments in CASCO. Springer Berlin Heidelberg, 1995.

[58] Vijay Vasandani and T. Govindaraj. Knowledge Structures for a Computer-Based Training Aid for Troubleshooting a Complex System. Springer Berlin Heidelberg, 1993.

[59] Joachim Wedekind. Computer-Aided Model Building. Springer US, United States, 1988.

[60] W.Feurzeig. Cognitive science, Artificial intelligence and complex training. Springer US, 1987.
[61] Randall Whitaker and Olov stberg. Channeling knowledge: Expert systems as communications media. Artificial intelligence review, 2:197-208, 1988. 